\title{
Beschränkte Therapieoptionen für eine häufige Erkrankung
}

Das Restless-Legs-Syndrom (RLS) erfährt in den letzten Jahren zunehmende Aufmerksamkeit. Für die Therapie des RLS stehen aktuell nur dopaminerge Substanzen (L-Dopa und Dopaminagonisten) zur Verfügung.

$\mathrm{Zu}$ den Problemen der dopaminergen Therapie zählte Professor Magdolna Hornyak, Algesiologikum/Zentrum für Schmerzmedizin, München, die nachlassende Wirkung am Morgen (End-of-Dose-Rebound) und Rebound-Phänomene bei Reduktion oder Absetzen der Medikation. Als schwerste Nebenwirkung dopaminerger Substanzen gilt die Augmentation, bei der gleichzeitig ein Wirkungsverlust der Therapie und eine $\mathrm{Zu}$ nahme der Symptomatik auftreten. PD
Dr. Ilonka Eisensehr, Bonomed Studienzentrum München, machte darauf aufmerksam, dass es unter der dopaminergen Medikation auch zu Blutdruckregulationsstörungen mit Hypotonie sowie zu Zwangs- und Suchtverhalten (z.B. Spielund Kaufsucht) kommen könne.

$\mathrm{Da}$ in vielen Fällen kein ausreichender Therapieerfolg zu erzielen sei, so Hornyak, muss der Arzt nicht zugelassene Medikamente - häufig Opioide und Antiepileptika - als Off-Label-Use verordnen. Niedrig dosierte Opioide hätten sich in der Praxis als „klinisch gut wirksam“ gezeigt. Für die Kombination aus redardiertem Oxycodon und Naloxon (Targin ${ }^{\circledR}$ ) zur Behandlung des RLS läuft aktuell ein Zulassungsverfahren.
Auf die aktuellen Leitlinien verwies Professor Svenja Happe, Klinik Maria Frieden, Telgte. Bei RLS-Patienten sei ein Ferritin-Zielwert von mindestens $50 \mu \mathrm{g} / \mathrm{l}$ definiert [http://www.awmf.org/leitlinien/detail/ll/030-081.html]. In der Praxis werden oft 50-100 $\mu \mathrm{g} / \mathrm{l}$ angestrebt. Ein Eisenmangel zähle zu den Auslösefaktoren für RLS und erhöhe das Augmentationsrisiko. In den Leitlinien wird auch auf weitere auslösende Faktoren einschließlich Medikamente wie Mirtazapin oder Citalopram verwiesen.

Dr. Andreas Fischer, freier Medizinjournalist

Pressekonferenz „RLS-Behandlung heute: Therapieziele erreicht?", München, 28.6.2013 Veranstalter: Mundipharma

\section{Zwei Jahre Fingolimod bei schubförmiger MS}

Wirkt die immunmodulierende Basistherapie bei MS unzureichend oder lässt ihre Wirkung nach, steht im Rahmen der sogenannten Eskalationstherapie seit zwei Jahren mit Fingolimod, dem ersten oralen MS-Therapeutikum, eine weitere effektive Option zur Verfügung.

Den ersten Durchbruch in der MS-Therapie brachten die Interferone. Dennoch ist ihr Effekt mit einer Reduktion der Schubrate um etwa $30 \%$ nach heutigen Maßstäben eher mäßig, erklärte Professor Hans-Peter Hartung, Direktor der neurologischen Universitätsklinik Düsseldorf. Mit modernen Second-Line-Medikamenten wie Fingolimod (Gilenya ${ }^{\varpi}$ ) lässt sich nicht nur die Schubrate deutlich effektiver senken. Auch die Progression der Behinderung und der Hirnatrophie können gebremst werden. Wie die Ergebnisse der placebokontrollierten zweijährigen FREEDOMS-Studie [Kappos L et al. New Engl J Med 2010; 362: 387 -401] und der Interferon-beta-1a-i.m.-kontrollierten einjährigen TRANSFORMS-
Studie [Cohen JA et al. New Engl J Med 2010; 362: 402 - 15] mit Fingolimod zeigen, ist die Marke der möglichen Schubreduktion auf über $50 \%$ gestiegen. Das Risiko für ein Fortschreiten des Behinderungsgrads war in der Fingolimod-Gruppe um $30 \%$ geringer, und auch das Risiko für einen Verlust von Hirnvolumen war signifikant niedriger.

Beide Studien wurden offen um ein weiteres Jahr verlängert, das heißt, bei $\mathrm{Pa}$ tienten der Fingolimod-Gruppe wurde die Therapie fortgesetzt und Patienten der Kontrollgruppen wurden auf Fingolimod umgestellt. Sowohl bezüglich der Schubrate als auch der Progression von Behinderung und Hirnatrophie waren die Patienten, die kontinuierlich Fingolimod erhalten hatten, im Vorteil.

„Dies spricht eindeutig dafür, dass Fingolimod umso effektiver ist, je früher es eingesetzt wird“, betonte Professor Tjalf Ziemssen, Leiter des Multiple Sklerose Zentrums am Zentrum für Klinische Neurowissenschaften, Dresden. Für die
Praxis ist jedoch die Zulassung als Second-line-Therapie relevant. Wie sich das erste orale Medikament in der täglichen Praxis bewährt, wird in der umfangreichen Registerstudie PANGAEA untersucht. Die ersten Zwischenergebnisse von 930 Patienten, für die Anfang 2013 mindestens ein Jahr Dokumentation vorlag [Ziemssen T et al. AAN-Jahrestagung, San Diego, Poster P01.185], zeigen, dass die Schubrate im ersten Jahr nach der Umstellung auf Fingolimod von 1,22 auf 0,33 (-73\%) abnahm - unabhängig von der Vorbehandlung. Etwa zwei Drittel der Patienten waren frei von Krankheitsaktivität. Den Therapieerfolg beurteilten $86 \%$ der Patienten und Ärzte als „gut“ bis „sehr gut“. Die gute Verträglichkeit von Fingolimod in klinischen Studien hat sich auch in der Praxis bestätigt.

Martin Bischoff, freier Medizinjournalist

Pressegespräch „Normal leben mit MS? - Therapie nachhaltig optimieren mit Gilenya ${ }^{\circledR}$, München, 16.5.2013; Veranstalter: Novartis Pharma 